\title{
Processing and characterization of chitosan microspheres to be used as templates for layer-by-layer assembly
}

\author{
Jessica M. R. Grech • João F. Mano • \\ Rui L. Reis
}

Received: 28 November 2007/Accepted: 9 March 2010/Published online: 3 April 2010

(C) Springer Science+Business Media, LLC 2010

\begin{abstract}
Chitosan (Ch) microspheres have been developed by precipitation method, cross-linked with glutaraldehyde and used as a template for layer-by-layer (LBL) deposition of two natural polyelectrolytes. Using a LBL methodology, Ch microspheres were alternately coated with hyaluronic acid (HA) and Ch under mild conditions. The roughness of the Ch-based crosslinked microspheres was characterized by atomic force microscopy (AFM). Morphological characterization was performed by environmental scanning electron microscopy (ESEM), scanning electron microscopy (SEM) and stereolight microscopy. The swelling behaviour of the microspheres demonstrated that the ones with more bilayers presented the highest water uptake and the uncoated cross-linked $\mathrm{Ch}$ microspheres showed the lowest uptake capability. Microspheres presented spherical shape with sizes ranging from 510 to $840 \mu \mathrm{m}$. ESEM demonstrated that a rougher surface with voids is formed in multilayered microspheres caused by the irregular stacking of the layers. A short term mechanical stability assay was also performed, showing that the LBL procedure with more than five bilayers of HA/Ch over $\mathrm{Ch}$ cross-linked microspheres provide higher mechanical stability.
\end{abstract}

J. M. R. Grech · J. F. Mano (凹) · R. L. Reis

Department of Polymer Engineering, 3B's Research Group-

Biomaterials, Biodegradables and Biomimetics, Campus de

Gualtar, 4710-057 Braga, Portugal

e-mail: jmano@dep.uminho.pt

URL: www.3bs.uminho.pt

J. M. R. Grech · J. F. Mano · R. L. Reis

IBB-Institute for Biotechnology and Bioengineering,

PT Government Associated Laboratory, Braga, Portugal

\section{Introduction}

Over the years, there has been an increasing interest in developing different forms of biomaterials to be applied in tissue engineering and drug delivery. Especially, particulate materials have been designed and optimized in various ways in order to improve patients care. So, there is a continuous effort in developing new methods, materials and devices. Microspheres have found a great applicability in tissue engineering because of their small size, being able to be inserted in the target area in a non-invasive manner; and large surface to volume ratios and a capacity for loading the bioactive agent at a high fraction of the total weight of the particle [1]. Such microspheres can be used to build scaffolds by simple agglomeration $[2,3]$ or they could be used as injectable scaffolds [4].

Chitosan (Ch) has been used in the particulate form for many purposes, including a carrier for drugs [5, 6]. Being a natural biodegradable biocompatible cationic polymer, it is used in large scale in the biomedical field. Because of its hydrophilic character, the water can penetrate in the polymer chains causing swelling, originating a hydrogel. These hydrogels are prevented from dissolving in the medium by chemical or physical crosslinking. For tissue engineering and drug delivery, hydrogels can either have the drug dissolved in the matrix or encapsulated. Also, the drug can be released upon swelling or by degradation of the hydrogel [7].

Since around 1990, when Decher et al. developed multilayer coatings based on the deposition of oppositely charged polyelectrolytes onto surfaces [8], the multilayered self-assembled or layer-by-layer (LBL) films have been applied in various areas, including biomaterials' coatings [9], which find great applicability in tissue engineering, biomimetic coatings [10], drug delivery [11], protein adsorption [12], bioactive coatings [13], biosensors and for 
the coating of living cells [14]. LBL architectures are based on the alternately deposition of oppositely charged species on any charged substrate [15], ranging from planar [16] to non-planar templates such as colloids [17] or microcapsules [11]. Since it is possible to use a wide variety of species and charged substrates and by varying the number of layers deposited [9], one can engineer materials by LBL construction with wide functional properties [18]. This technique becomes very relevant for biomedical applicability since it can incorporate different types of biomolecules in or on the materials. The LBL construction also enables to control the thickness of the layers at a nanometer scale and produce robust coatings under ambient and physiological conditions due to strong multiple electrostatic interaction forces between layers [19]. Multilayered self-assembled methodology is, consequently, a versatile, easy preparation technique with small costs involved. However, the biggest limitation might be the time-consuming sequential polyelectrolyte deposition cycles and purification steps [20].

Also, it is possible to tune the degradability of the LBL films [16] by using biodegradable polymers in the LBL architecture. Natural polyelectrolytes have been used in such technology, including a series of polysaccharides, e.g. alginate [21, 22], Ch [12, 23], hyaluronic acid (HA) [12, 23], dextran sulphate [21] and their combinations. Among these, $\mathrm{Ch}$ and HA have been widely applied as biomedical materials in various forms. Up to now these natural polymers have been used as sponges [24], films $[25,26]$, capsules [27] or other hydrogel or membrane form. In this work, we hypothesize that the LBL methodology could be used to coat $\mathrm{Ch}$ microspheres in order to broaden the versatility of the use of such systems in different biomedical applications. For example, in the context of tissue engineering, the microspheres could act simultaneously as a support for cell adhesion/proliferation and as a matrix to release relevant bioactive agents, including signalling molecules to control cell functions. Here, the LBL coating could act as a transport barrier, enabling to tune the release profile of bioactive agents, or as a substrate to control protein adsorption and cell attachment.

In this work, the microspheres were characterized morphologically by stereolight microscopy, scanning electron microscopy (SEM) and environmental scanning electron microscopy (ESEM). Swelling behaviour and a short term mechanical stability assay were also performed.

\section{Materials and methods}

\subsection{Materials}

Medium molecular weight $\mathrm{Ch}$ (degree of deacetylation of $83.86 \%$, determined by $\mathrm{H}$ NMR, and molecular

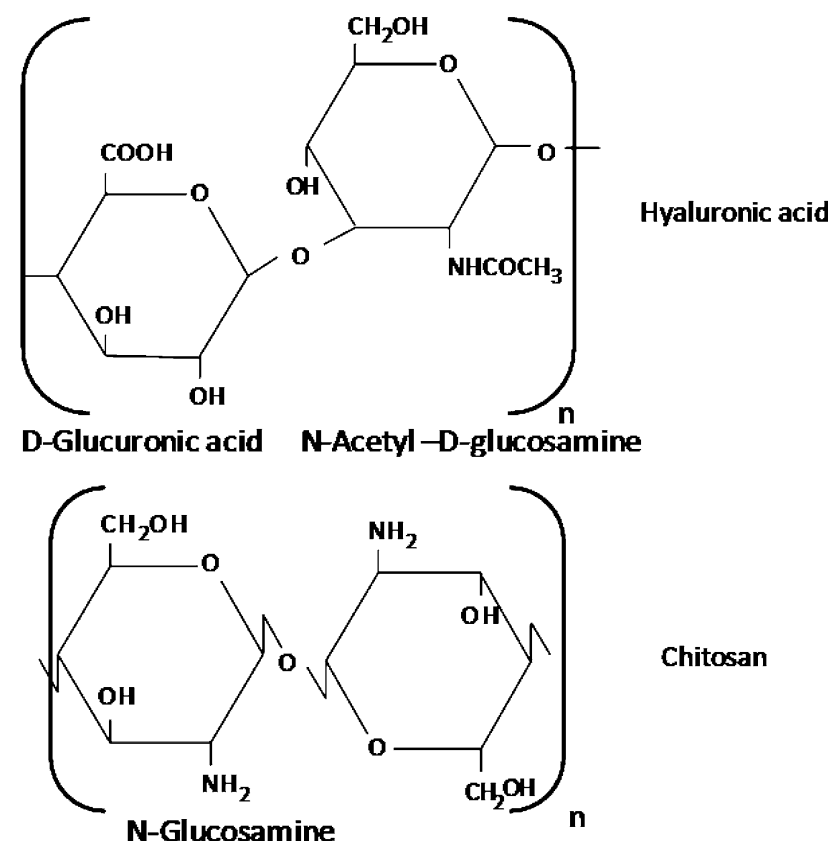

Fig. 1 Molecular structure of both chitosan and hyaluronic acid used in this work

viscosimetric weight of $662 \mathrm{kDa}$ ) and glutaraldehyde (Glu) were purchased from Sigma-Aldrich. HA sodium salt from Streptococcus equi. sp. (HA) with an average molecular weight of $1.63 \times 10^{6} \mathrm{Da}$ was obtained from BioChemika Fluka. Figure 1 illustrates the molecular structure of both $\mathrm{Ch}$ and HA. Acetic acid glacial $\left(\mathrm{CH}_{3} \mathrm{COOH}\right)$, sodium hydroxide pellets $(\mathrm{NaOH})$ and sodium chloride $(\mathrm{NaCl})$ were purchased from Panreac Quimica SA.

\subsection{Preparation of chitosan microspheres and further crosslinking reactions}

A $1.5 \%(w / v)$ Ch solution was prepared by dissolving $\mathrm{Ch}$ overnight in a $2 \%$ acetic acid solution. Further filtration by vacuum was carried out, using a nylon filter and a Milipore membrane $(5 \mu \mathrm{m})$ was carried out to remove the sludges from this natural polymer.

Microspheres were prepared by a precipitation method, based on Dini et al. [28] and Malafaya et al. [2] with minor modifications. The $\mathrm{Ch}$ solution was extruded through a $0.3 \mathrm{~mm}$ needle (BD-Microfine U-100 insulin) at a constant rate of $4 \mathrm{ml} / \mathrm{h}$ using a syringe pump (World Precision Instruments, USA), and $\mathrm{Ch}$ microspheres were formed once the droplets reached a $1 \mathrm{M} \mathrm{NaOH}$ solution from a height of $5 \mathrm{~cm}$, under continuous stirring. After the formed microspheres, the agitation continued for $2 \mathrm{~h}$ to guaranty complete precipitation. Subsequently, the microspheres (referred as A) were removed from the precipitation bath and consistently washed with distilled water. 
Further cross-linking of the microspheres was performed by immersing them in a $0.025 \mathrm{M}$ Glu solution for $30 \mathrm{~min}$. The cross-linked $\mathrm{Ch}$ microspheres (A30) were repeatedly washed with distilled water to remove the excess of the crosslinking agent. The microspheres were kept in distilled water until further use.

\subsection{Roughness of the $\mathrm{Ch}$ microspheres by atomic force microscopy}

A Multimode Digital Instruments (from LabMatUniversity of Minho) atomic force microscope was used to observe the surface of A and A30, in the dry state and at room temperature. In the case of dry state, samples were dehydrated in different increasing concentrations of ethanol $(25,30,50,70,80,90$ and $100 \%)$, for $30 \mathrm{~min}$ each, and set to dry at room temperature. The observation was performed in the tapping mode using tips model RTESP (Veeco) at room temperature and with a scan rate of $1.001 \mathrm{~Hz}$.

\subsection{Polyelectrolyte LBL deposition}

$0.5 \%$ (w/w) HA solution was mixed with $0.15 \mathrm{M} \mathrm{NaCl}$ in distilled water and stirred overnight at room temperature, in order to hydrate the polymer. $1.5 \%$ (w/w) Ch solution was also prepared following the same procedure described for the HA solution. The final $\mathrm{pH}$ of both solutions was adjusted to 4.5 with $0.1 \mathrm{M} \mathrm{CH} \mathrm{CH}_{3} \mathrm{COOH}$ or $1 \mathrm{M} \mathrm{NaOH}$, allowing the solutions to dissolve and also make $\mathrm{Ch}$ positively charged and HA negatively charged. The polyelectrolyte multilayer deposition assembly on the microspheres was then accomplished by the consecutive adsorption of the oppositely charged polyelectrolytes with rinsing steps with a solution of $0.15 \mathrm{M} \mathrm{NaCl}$ ( $\mathrm{pH}$ adjusted to 4.5) between adsorption layers. Each deposition took $15 \mathrm{~min}$ and the rinsing steps about 2 min [29]. 3, 5, 8 and 10 bilayers $(\mathrm{M} 3 \times, \mathrm{M} 5 \times, \mathrm{M} 8 \times$ and $\mathrm{M} 10 \times$, respectively) were performed on the A30 microspheres. After the multilayer construction, the microspheres were washed with distilled water. Multilayer microspheres were kept in distilled water, until further use. Figure 2 represents a scheme of the LBL procedure used in this work.

\subsection{Swelling behaviour of the multilayered microspheres}

Dried microspheres with a known weight were placed in $10 \mathrm{ml}$ of a $\mathrm{pH} 7.4$ phosphate buffer saline solution and allowed to swell at $37^{\circ} \mathrm{C}$ for pre-determined times $(15,30$, 45, 60, 120, 240, 360, 480, 1440 and $4320 \mathrm{~min})$. Triplicate samples were used for each test period.
At the pre-determined time, the microspheres were removed from the solution, and the wet weight of the microspheres was determined by blotting them with filter papers to remove the excess of solution on the surface and weighed immediately. The accuracy of the balance used was $0.0001 \mathrm{~g}$. The percentage of degree of swelling was calculated according to Eq. 1:

Degree of swelling $(\%)=\left[\left(m_{t}-m_{i}\right) /\left(m_{i}\right)\right] \times 100$

where $m_{t}$ and $m_{i}$ are the weight of the sample at the specific test time $t$ after absorbing the excess of solution and in the dry state, respectively.

\subsection{Determination of size and morphology}

The size and morphology of the microspheres were investigated by stereomicroscopy (Zeiss-Stemi 2000-C KL 1500 LCD, 459315). Also, scanning electron microscopy, SEM (Leica Cambridge S360), and environmental scanning microscopy, ESEM (FEI Quanta 400 with EDS), were used to study the morphology of the microspheres.

Both stereomicroscope and ESEM were used to evaluate the morphology of the microspheres in their swollen form (distilled water), while for SEM the samples were first dehydrated in different increased concentration series of ethanol (25, 30, 50, 70, 80, 90, and 100\%, 30 min each), dried at room temperature, mounted on metal stubs using double sided conducting adhesive tape and vacuum coated with gold in a sputter coating unit. Fifteen microspheres in the swollen state were measured for each sample, while in the dried state, an average of eight microspheres were measured under SEM.

\subsection{Assessment of the mechanical stability}

A short term stability assay was carried out to study the behaviour of the different microspheres submitted to a combination of destabilising forces as to mimic the physiological conditions. The procedure was based on Orive et al. [30, 31] with some modifications. Briefly, Ch microspheres were distributed in five well plates with 10 microspheres in each well. This same procedure was done for $\mathrm{A} 30, \mathrm{M} 5 \times$ and $\mathrm{M} 10 \times$ microspheres. In each well, $800 \mu \mathrm{l}$ of phosphate buffer solution (PBS) was added and each culture plate was placed in a shaker at $200 \mathrm{rpm}$ and $37^{\circ} \mathrm{C}$. At 1, 3, 6, 24 and $48 \mathrm{~h}$, ruptured microspheres from each well were counted with a stereolight microscope for statistical evaluation. $48 \mathrm{~h}$ was the maximum time period which corresponded to previous experimental work on total drug release, using the same material [32]. Results are presented as the percentage of ruptured microspheres as a function of time. 
Fig. 2 Scheme of the electrostatic layer-by-layer polyelectrolyte deposition

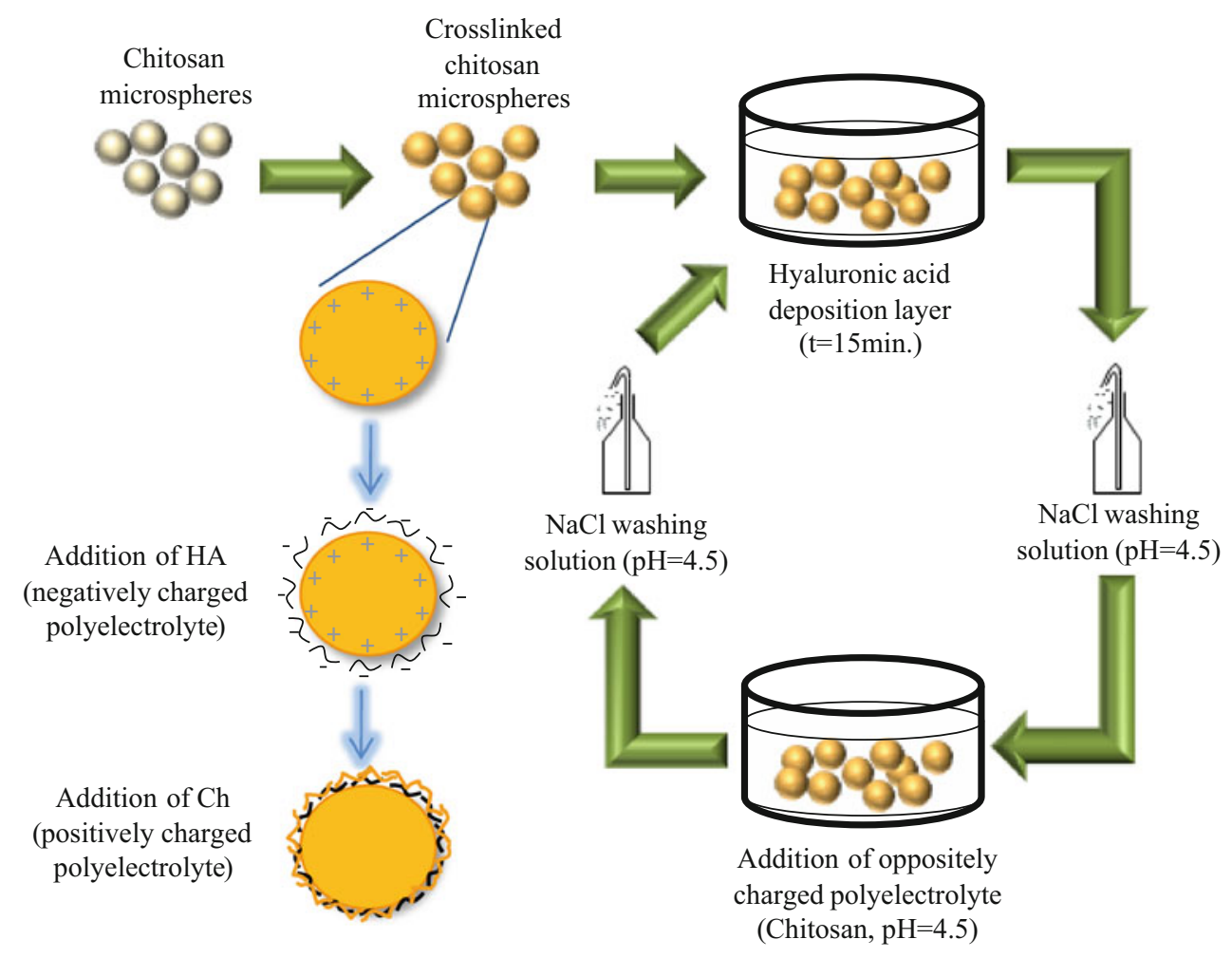

\section{Results and discussion}

Ch microspheres were developed by a precipitation method and further cross-linked with glutaraldehyde $(0.025 \mathrm{M})$. These microspheres were used as a substrate for LBL deposition of HA and Ch. HA and Ch were prepared in a $\mathrm{NaCl}$ solution. $\mathrm{NaCl}$ solution used for the preparation of the polyelectrolytes and for the rinsing solution played an important role on the final morphology of the multilayered microspheres. This fact has been studied before by several researchers, namely Mo et al. [33], Kim et al. [34], and Ye et al. [35], who confirm that by using polyelectrolyte solutions prepared in a high concentration of $\mathrm{NaCl}$ solution, such as $0.15 \mathrm{M}$, during the LBL procedure it seems to favour a rougher surface upon an increase in the number of layers.

The multilayer microspheres are thought to be useful in tissue engineering, for example, as a support for cell adhesion/proliferation or as a matrix to release bioactive agents; the LBL coating could also be meant to act as a transport barrier, tuning their release. Besides behaving as a matrix, the multilayer microspheres could also be incorporated in a scaffold, releasing the agent while the surrounding material degrades. Therefore, LBL methodology on $\mathrm{Ch}$ microspheres might be a valuable procedure in the biomedical field, especially in controlled drug release and regenerative medicine.
3.1 Determination of the roughness of cross-linked and uncross-linked $\mathrm{Ch}$ microspheres by atomic force microscopy

Figure 3 evidences a clear difference in the surface between uncross-linked $\mathrm{Ch}$ microspheres (A) and crosslinked $\mathrm{Ch}$ microspheres (A30). A30 has a rougher surface compared to A, probably due to the crosslinking effect, since both materials only differ in the presence of glutaraldehyde. The average roughness determined for microspheres A was $26.1 \mathrm{~nm}$ while A30 presented an average of $58.8 \mathrm{~nm}$, more than twice compared to A.

\subsection{Swelling behaviour}

In vitro swelling studies were conducted in PBS (pH 7.4) at $37^{\circ} \mathrm{C}$. The swelling degree for the different microspheres was determined by a gravimetric method.

The percentage of swelling degree in function of time is given in Fig. 4 depending on the type of microsphere, all cross-linked with $0.025 \mathrm{M}$ glutaraldehyde. As observed, there is a difference between the swelling degree in microspheres that have not been cross-linked (A) and glutaraldehyde cross-linked microspheres $(\mathrm{A} 30, \mathrm{M} 5 \times, \mathrm{M} 10 \times)$. The degree of swelling in microspheres has shown a decreasing trend upon crosslinking with glutaraldehyde: microspheres A show a maximum swelling degree of $132 \%$ within a period 
Fig. 3 Roughness of chitosan microspheres A (a1 and $\mathbf{a} 2)$ and cross-linked chitosan microspheres A30 (b1 and b2) determined by atomic force microscopy
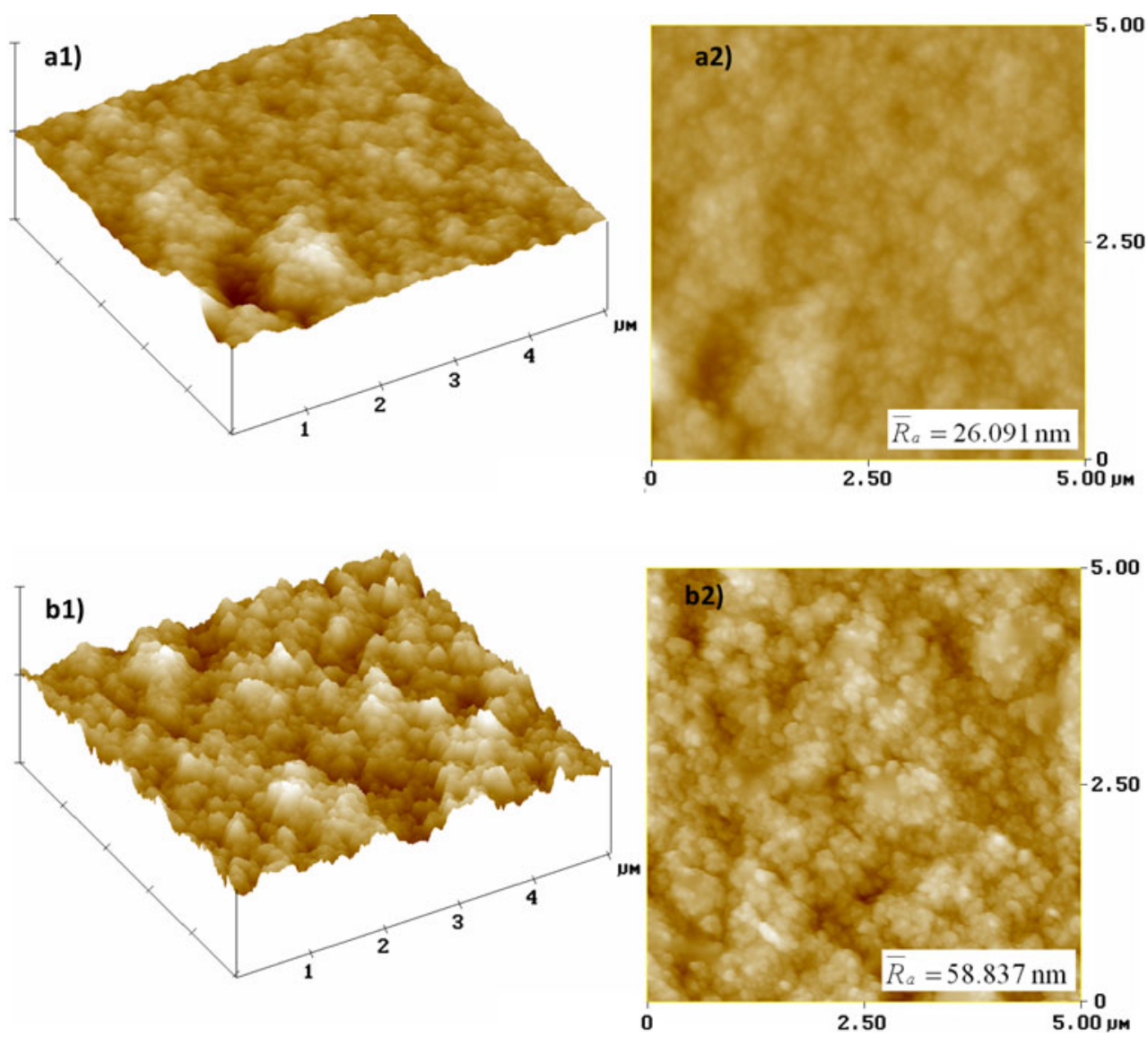

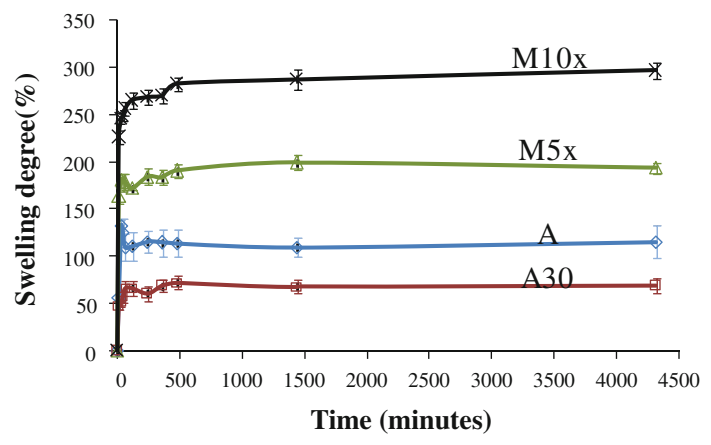

Fig. 4 Swelling behaviour of chitosan microspheres and multilayered microspheres as a function of time, at $\mathrm{pH} 7.4$ and $37^{\circ} \mathrm{C}$

of $30 \mathrm{~min}$, despite only reaching equilibrium of $115 \%$ at 240 min; microspheres A30 reach a maximum swelling degree of $72 \%$ within 480 min maintaining the equilibrium for the following test period. The effect of the swelling in hydrogels has also been observed by Gupta and Kumar [36] and Gupta and Jabrail [37]. This swelling behaviour can be explained by the fewer water molecules capable of incorporating the $\mathrm{Ch}$ chain due to the entanglement of the chain caused by glutaraldehyde, impeding, therefore, to have higher swelling rate than $\mathrm{A}$, which do not have chemically cross-linked chains and water penetrates more freely.
Multilayered microspheres present more hydrophilic groups in their chains $(\mathrm{M} 10 \times$ followed by $\mathrm{M} 5 \times$ ), giving rise to higher degrees of swelling. As can be seen from Fig. 4, M5× and M10× reach equilibrium of 199 and $286 \%$ within $1440 \mathrm{~min}$, respectively. These high values of swelling degree for $\mathrm{M} 5 \times$ and $\mathrm{M} 10 \times$ microspheres, as compared with the uncoated ones, might be explained based on the fact that HA is a hydrophilic charged polymer [38]. Since M10× has more layers than M5×, it would uptake more water than the latter. Despite the fact that the multilayers had been crosslinked after their development, the effect of the hydrophilicity of HA seems to overcome the effect of crosslinking with $0.025 \mathrm{M}$ Glu.

The changes in the swelling behaviour of the system could be also triggered by the effect of external variables [39], such as temperature, by incorporating, for example, thermo-responsive polymers in the particles [40, 41].

\subsection{Microspheres size and morphology}

The size and morphology of the developed microspheres were analysed by stereolight microscopy, SEM and ESEM and the images are presented in Figs. 5, 6, 7 and 8. It is possible to see that the different microspheres possess an approximately spherical shape but vary in size and 
morphology (see Table 1 and Fig. 6). Multilayered microspheres showed a size of about $1700 \mu \mathrm{m}$ when swollen and around 770-840 $\mu \mathrm{m}$ when dried, as shown in Table 1. All microspheres developed exhibit a spherical shape and under stereolight microscopy, the microspheres appeared to have good stability in water (Fig. 5). In the dried state, there is a slight decreasing size from $\mathrm{M} 5 \times$ to $\mathrm{M} 8 \times$. However, the standard deviation is higher for M $8 \times$ than for $\mathrm{M} 5 \times$, which means that there is a higher dispersion of the microspheres size comparatively to M5 $\times$, in respect to the mean value. In the case of the multilayered microspheres size in the swollen state, the microspheres were measured in a stereolight microscopy, which has a reduced depth of focus and resolving capacity then other characterization techniques capable of observing samples in the humid state. The similar sizes of M5 $\times, M 8 \times$ and M10 $\times$ might not have been measured precisely and accurately, and as it can be confirmed by the high values of standard deviations. Stereolight microscopy demonstrated to be a useful technique to also observe the shape of the microspheres. Also relating the swelling behaviour with the microsphere size of A and A30, from Fig. 4 it is observed that A has a higher swelling degree than A30, which was expected since when crosslinking occurs, fewer molecules of water are capable of incorporating the $\mathrm{Ch}$ chain due to the entanglement of the chain caused by the crosslinker.
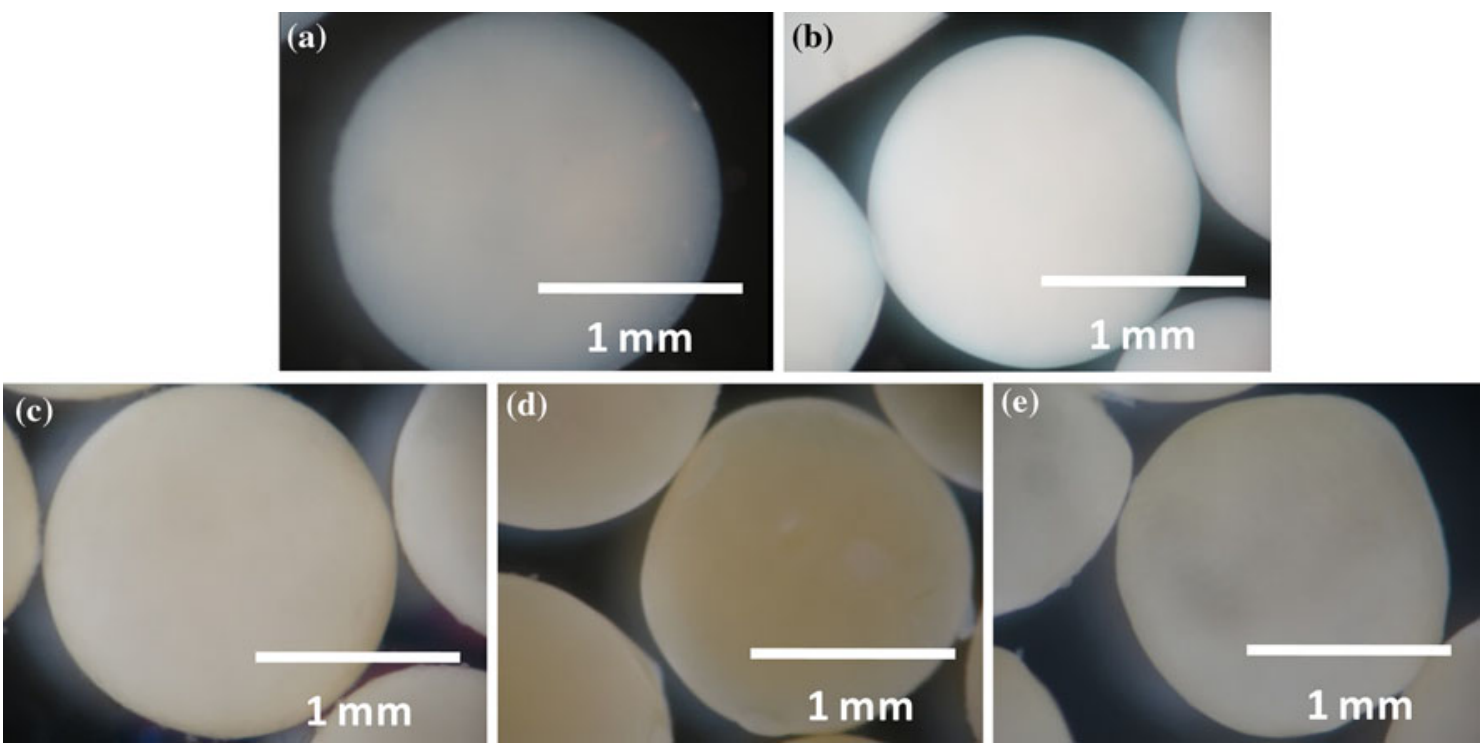

Fig. 5 Stereolight microscopy images of chitosan microspheres and multilayered microspheres. a Chitosan microspheres (A), b crosslinked chitosan microspheres (A30), c $(\mathrm{HA} / \mathrm{Ch})_{5}$ multilayered

microspheres $(\mathrm{M} 5 \times), \quad$ d $(\mathrm{HA} / \mathrm{Ch})_{8}$ multilayered microspheres $(\mathrm{M} 8 \times)$, and e $(\mathrm{HA} / \mathrm{Ch})_{10}$ multilayered microspheres $(\mathrm{M} 10 \times)$
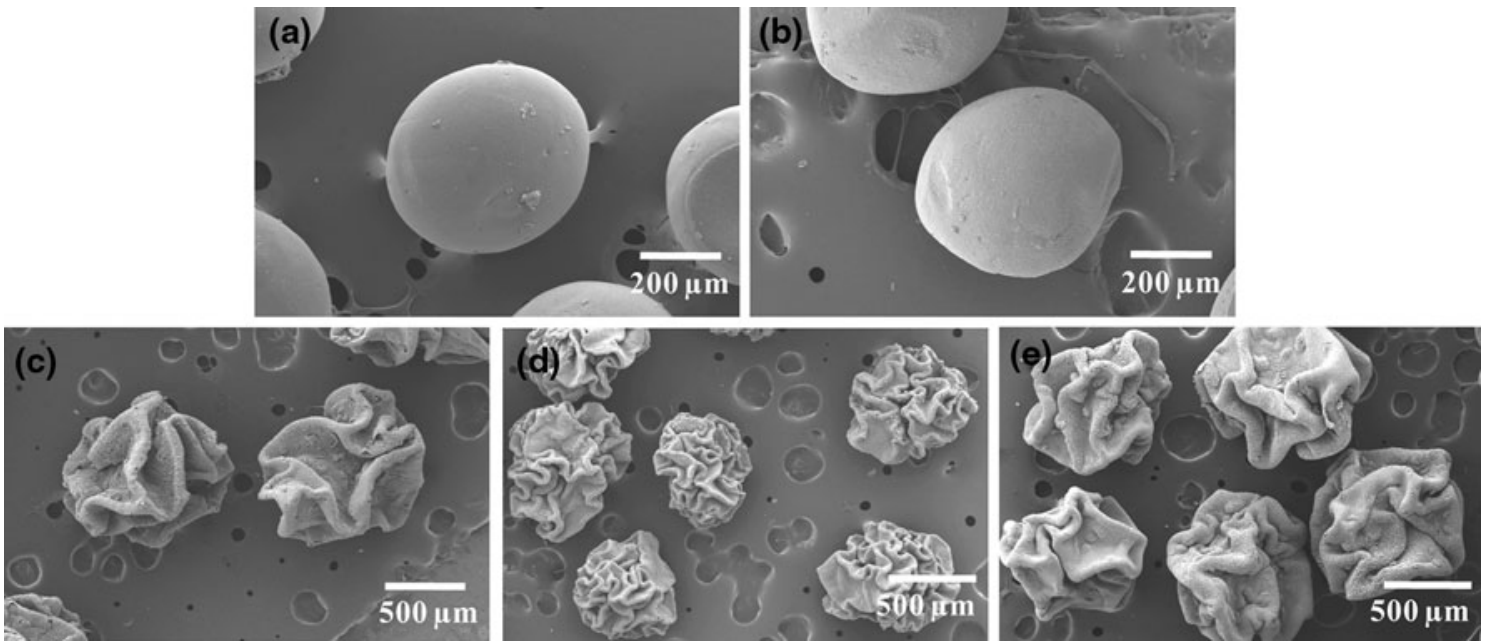

Fig. 6 Scanning electron microscopy images of chitosan microspheres and multilayered microspheres. a A, b A30, c M5×, d M8× and e M10× 

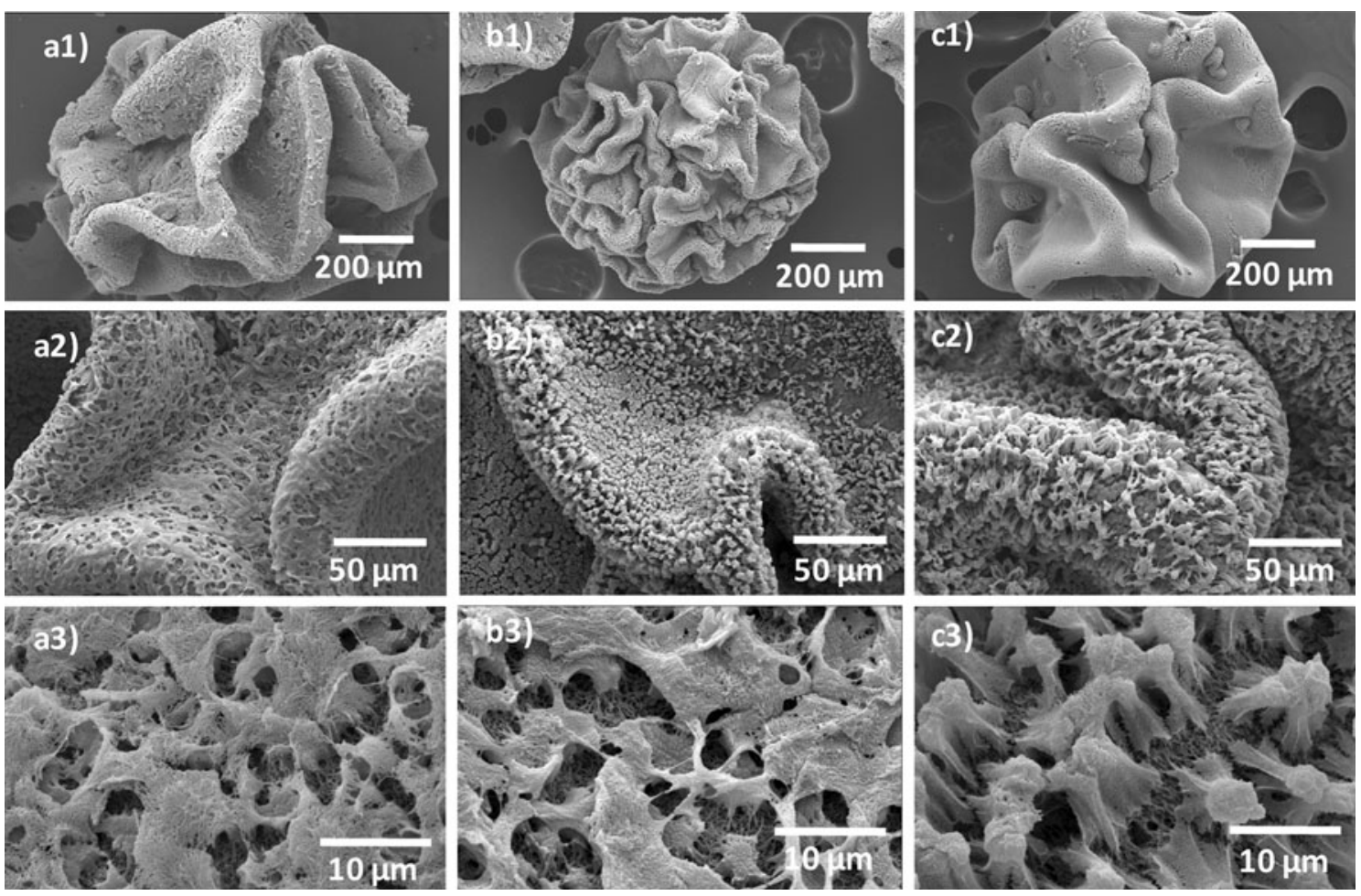

Fig. 7 Scanning electron microscopy images of multilayered microspheres. a1-a3 M5×, b1-b3 M8× and c1-c3 M10×
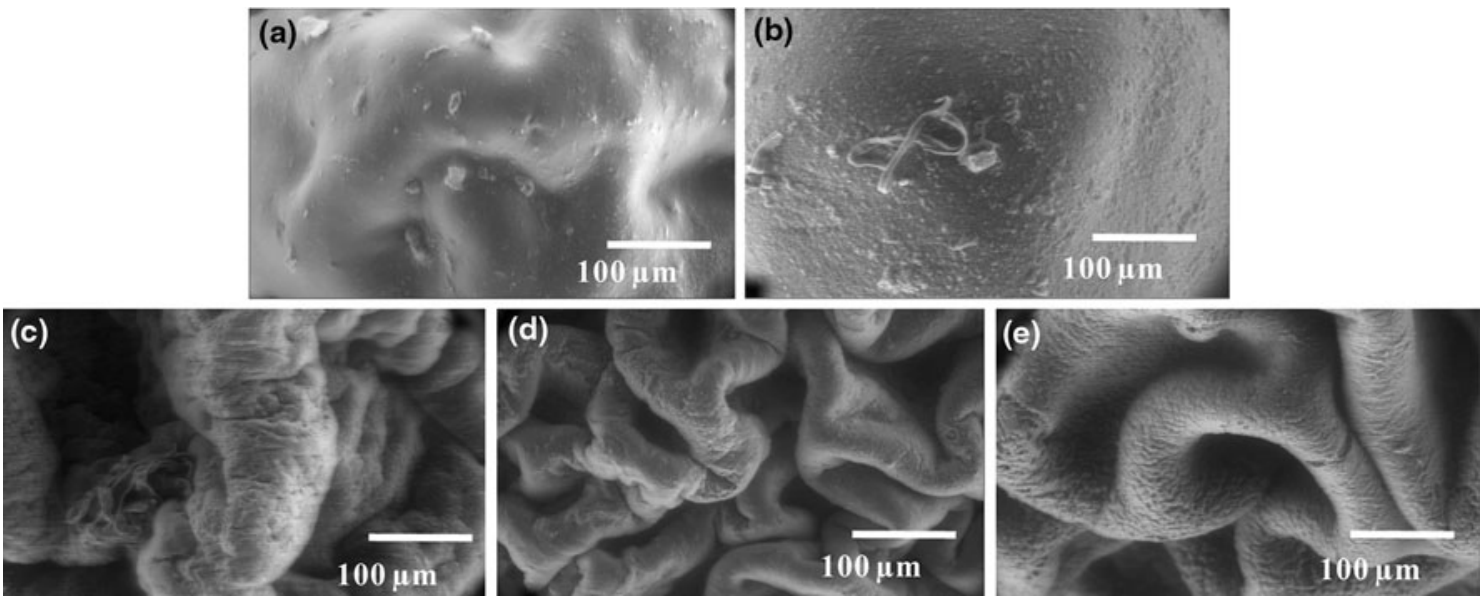

Fig. 8 Environmental scanning electron microscopy images of chitosan microspheres and multilayered microspheres. a A, b A30, c M5×, d $\mathrm{M} 8 \times$ and e M10x

Table 1 Average size of the chitosan microspheres and multilayered microspheres developed (mean \pm standard deviation); $n=15$ microspheres (swollen microspheres); $n=8$ microspheres (dried microspheres)

\begin{tabular}{lll}
\hline Microspheres & $\begin{array}{l}\text { Swollen state }(\mu \mathrm{m})(\text { detected by } \\
\text { stereolight microscopy })\end{array}$ & $\begin{array}{l}\text { Dried state }(\mu \mathrm{m}) \\
(\text { detected by SEM) }\end{array}$ \\
\hline A & $1800 \pm 65.46$ & $510 \pm 6.78$ \\
A30 & $1600 \pm 25.82$ & $510 \pm 5.35$ \\
M5 & $1700 \pm 65.46$ & $800 \pm 5.17$ \\
M8 & $1700 \pm 59.36$ & $770 \pm 10.69$ \\
M10 & $1700 \pm 45.77$ & $840 \pm 7.56$ \\
\hline
\end{tabular}


When crosslinking occurs, A30 does not allow water to penetrate as much as A. Therefore, the size is lower and the swelling degree is lower, comparatively to A.

SEM images (Fig. 6a, b) demonstrate a rather smooth surface in the case of the uncross-linked (A) and crosslinked (A30) dried Ch microspheres, compared to multilayered $\mathrm{Ch} / \mathrm{HA}$ dried microspheres $(\mathrm{M} 5 \times, \mathrm{M} 8 \times$ and M10 $\times$, Fig. $6 c-e$, respectively), which present a rougher surface. This could be the result of several effects. The overall roughness (Fig. 7a1, b1, c1) might be explained by the acidic environment of the deposition solutions and rinsing solution. In such conditions, the $\mathrm{Ch}$ microspheres are in a highly hydrated state as the amine groups are protonated. When the coated microspheres are washed in neutral solutions the microspheres tend to deswell, but not homogeneously due to the creasing effect caused by the coating. Another explanation may be related to the fact that during the LBL deposition the microspheres are immersed in $\mathrm{NaCl}$-containing solutions and swell in these liquids with high ionic strength; during washing in pure water the water inside the particles comes out by osmosis. This process may be fast and may induce the formation of a rough surface. With regard to the nano-roughness observed in Fig. 7a2, b2 and c2, this could be due to sodium ions that shield the electrostatic repulsion of HA molecules, and the molecules do not diffuse in and out unlike $\mathrm{Ch}$ molecules [42]. Richert et al. [42] also reported the importance in ionic strength higher than $0.15 \mathrm{M} \mathrm{NaCl}$ in the built up of $\mathrm{Ch}$ and HA layers, increasing therefore the total amount of both polyelectrolytes deposited after each layer. The promotion of thicker multilayers by the presence of salt has been reported by Kato et al. [43]. The irregular stacking of oppositely charged $\mathrm{Ch}$ and HA chains on the surface of the microsphere and the complexation between the polyelectrolytes multilayers could also explain the roughness presented, which has been reported by Ye et al. [11,35].

As expected, the A30 microspheres are slightly smaller than the A ones (see Table 1) in the swollen state, being in agreement with the swelling data.

From Fig. 7, SEM images demonstrate the morphology of the surface of the multilayered microspheres (M5 $\times$, $\mathrm{M} 8 \times$, and $\mathrm{M} 10 \times$ ). It is possible to detect the differences in the surface roughness of the microspheres and the formation of a dense packing on the surface [29]. As the number of HA/Ch polyelectrolyte layers increases $(\mathrm{M} 5 \times, \mathrm{M} 8 \times$ and $M 10 \times)$, the differences in the surface roughness of the microspheres are more pronounced as compared to the uncoated microspheres. Also, the "wavy" morphology on samples M $5 \times, M 8 \times$ and $\mathrm{M} 10 \times$, is also caused by the irregular stacking of polysaccharides on previous layers, as discussed by Ye et al. [11]. Also, ESEM images (Fig. 8) obtained in wet state confirm the "wavy" morphology of the multilayered microspheres observed in SEM.
The "wavy" morphology also plays important roles in the swelling capability. Because the surface of the microspheres have the wavy shape, more water is able to penetrate than a rather smooth surface (which is the case of A and A30, having lower swelling degrees compared to M5 $\times, M 8 \times$ and M10×). Microspheres A absorb more water in the first hours than that corresponding to their equilibrium swelling degree because of the need for the chains to stabilize until they reach the equilibrium, as all the samples tested.

\subsection{Assessment of the mechanical stability}

Microspheres were exposed to both agitation and osmotic swelling pressure to evaluate the mechanical strength under physiological conditions. Mechanical stability studies were performed on microspheres under agitation $(200 \mathrm{rpm})$, in PBS, for different periods of time, to the different developed formulations. Figure 9 shows some photographs where the typical breakage caused to the microspheres can be observed (see arrows). Only A, A30 and M3× presented some extent of rupture after $48 \mathrm{~h}$ of assessment, indicating that a LBL procedure with more than five bilayers of HA/ $\mathrm{Ch}$ over Ch cross-linked microspheres, provides higher mechanical resistance.

The percentage of ruptured microspheres as a function of time is given in Fig. 10. It is demonstrated that, by the end of the $48 \mathrm{~h}$ test period, microspheres $\mathrm{A}$ have the highest fraction of ruptured microspheres (14\%), followed by $\mathrm{A} 30$ and $\mathrm{M} 3 \times$, with 8 and $6 \%$ ruptured microspheres, respectively. Microspheres $\mathrm{M} 5 \times, \mathrm{M} 8 \times$ and $\mathrm{M} 10 \times \mathrm{did}$ not show, in the time periods tested, any rupture. These results indicate that multilayered cross-linked microspheres with more than three bilayers are mechanically more resistant than uncross-linked and cross-linked $\mathrm{Ch}$ microspheres.

The breakage of the microspheres under mechanical assessment is caused by the swelling effect encouraged by the mass transfer from the PBS solution through the microsphere surface [44]. Crosslinking promotes some mechanical stability in the microspheres. This is shown in Fig. 10, where all microspheres except $\mathrm{A}$, which are not cross-linked have less percentage of rupture, i.e., fewer water molecules penetrated inside the microsphere inducing the breakage of the membrane of the microspheres by osmosis. Microspheres A have a less stable structure compared to cross-linked microspheres.

Despite the fact that crosslinking may increase the resistance against mechanical rupture of the microspheres, fact evidenced by the lower percentage of ruptured crosslinked Ch microspheres (A30) over uncross-linked microspheres (A), it seems that LBL procedure on cross-linked Ch microspheres also increases their mechanical resistance, 

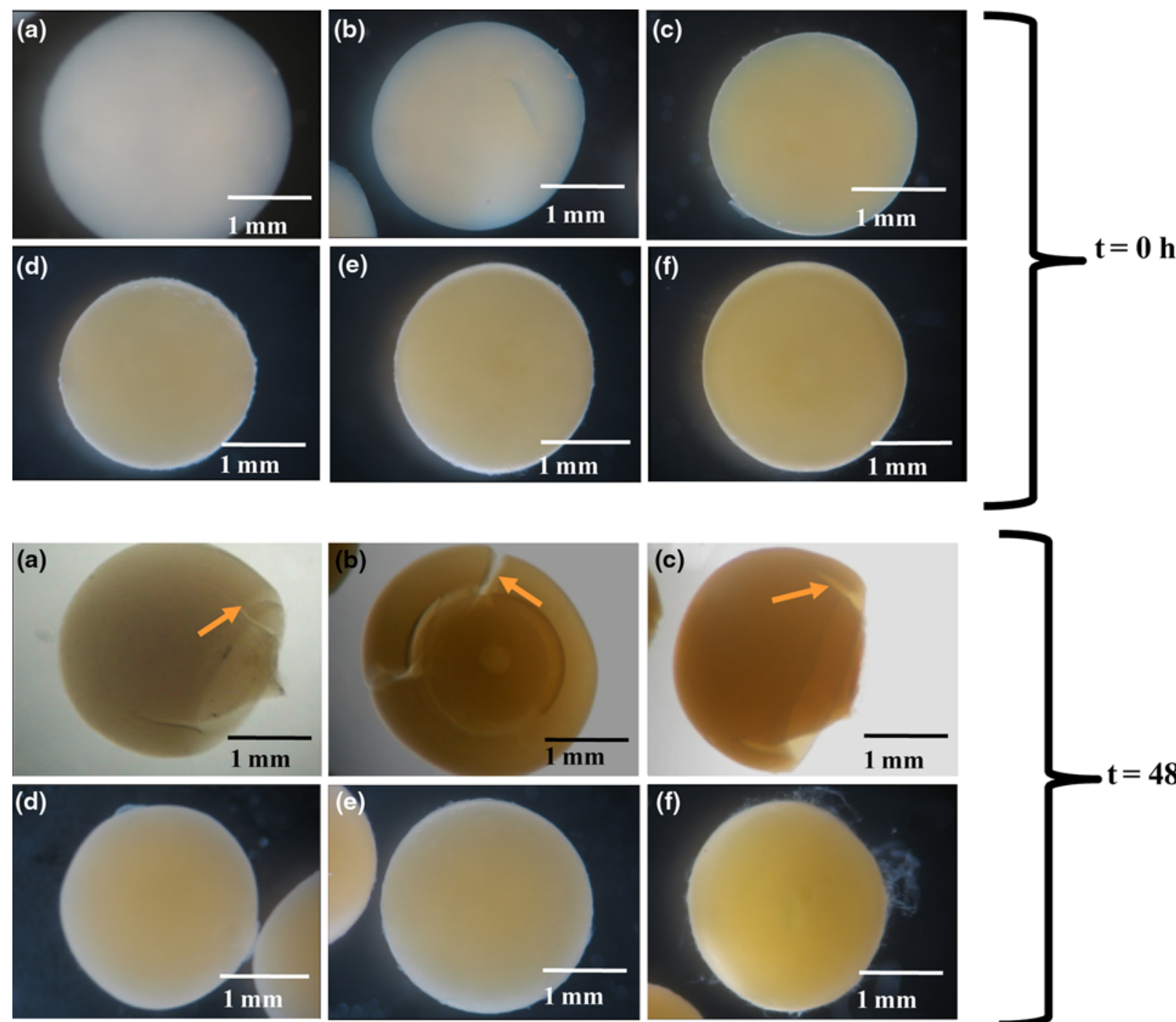

$\mathrm{t}=\mathbf{4 8 ~ h}$

Fig. 9 Photographs taken before (top) and after (down) the short-term mechanical assessment. a A, b A30, c M3×, d M5×, e M $8 \times$ and f M10×

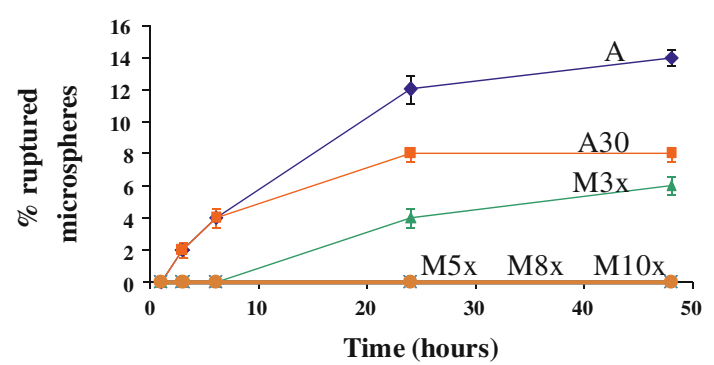

Fig. 10 Percentage of ruptured microspheres as a function of time, in PBS and at $37^{\circ} \mathrm{C}$

most likely because of the complexation between the oppositely charged polyelectrolytes, Ch and HA. Therefore, the multilayers seem to have a positive effect on the mechanical resistance of $\mathrm{Ch}$ cross-linked microspheres.
Therefore, resistance should be a result of the robust and stable coating that was built on the microspheres.

\section{Conclusions}

In conclusion, we have successfully built polyelectrolytes multilayers on $\mathrm{Ch}$ crosslinked microspheres including also $\mathrm{Ch}$ as a component of the coating, which could possibly be applied as a support for cell adhesion/proliferation and even as a matrix to release a bioactive agent. Ch-based cross-linked microspheres were prepared by a precipitation method and were used as a template for $\mathrm{Ch} / \mathrm{HA}$ multilayer deposition. The multilayer microspheres demonstrated good mechanical stability and higher swelling behaviour than single microspheres, possibly indicating that these 
types of microspheres might present higher loading of a bioactive agent or cells than simple microspheres. It is believed that such coated microspheres could extend the applicability of $\mathrm{Ch}$ microspheres in areas such as tissue engineering and in systems for the release of bioactive molecules. Particulate systems provide a suitable form of delivering a certain bioactive agent due to their unique size, surface area, and physicochemical properties. Therefore, it is believed that the LBL methodology on microspheres might create a sustained release of a possible bioactive agent from the microspheres.

Acknowledgments We gratefully acknowledge the funding from the European Union STREP HIPPOCRATES (NMP3-CT-2003505758), the European NoE EXPERTISSUES (NMP3-CT-2004500283) and the INTERREG III A Project SP1 P151/03, PROTEUS.

\section{References}

1. Silva GA, Ducheyne P, Reis RL. Materials in particulate form for tissue engineering. Basic concepts. J Tissue Eng Regen Med. 2007;1:4-24.

2. Malafaya PB, Pedro AJ, Peterbauer A, Gabriel C, Redl H, Reis RL. Chitosan particles agglomerated scaffolds for cartilage and osteochondral tissue engineering approaches with adipose tissue derived stem cells. J Mater Sci Mater Med. 2005;16:1077-85.

3. Borden M, Attawia M, Laurencin CT. The sintered microsphere matrix for bone tissue engineering: in vitro osteoconductivity studies. J Biomed Mater Res. 2002;61:421-9.

4. Cruz DMG, Ivirico JLE, Gomes ME, Ribelles JL, Sánchez MS, Reis RL, et al. Chitosan microparticles as injectable scaffolds for tissue engineering. Tissue Eng Reg Med. 2008;2:378-80.

5. Sinha VR, Singla AK, Wadhawan S, Kaushik R, Kumria R, Bansal K, et al. Chitosan microspheres as a potential carrier for drugs. Int J Pharm. 2004;274:1-33.

6. Prabaharan M, Mano JF. Chitosan-based particles as controlled drug delivery systems. Drug Deliv. 2005;12:41-57.

7. Peppas NA, Hilt JZ, Khademhosseini A, Langer R. Hydrogels in biology and medicine: from molecular principles to bionanotechnology. Adv Mater. 2006;18:1345-60.

8. Decher G, Hong JD, Schmitt J. Buildup of ultrathin multilayer films by a self-assembly process: III. Consecutively alternating adsorption of anionic and cationic polyelectrolytes on charged surfaces. Thin Solid Films. 1992;210:831-5.

9. Tang ZY, Wang Y, Podsiadlo P, Kotov NA. Biomedical applications of layer-by-layer assembly: from biomimetics to tissue engineering. Adv Mater. 2006;18:3203-24.

10. Zu XL, Lu ZQ, Zhang Z, Schenkman JB, Rusling JF. Electroenzyme-catalyzed oxidation of styrene and cis-beta-methylstyrene using thin films of cytochrome P450cam and myoglobin. Langmuir. 1999;15:7372-7.

11. Ye SQ, Wang CY, Liu XX, Tong Z, Ren B, Zeng F. New loading process and release properties of insulin from polysaccharide microcapsules fabricated through layer-by-layer assembly. J Control Release. 2006;112:79-87.

12. $\mathrm{Lu} \mathrm{HY,} \mathrm{Hu} \mathrm{NF.} \mathrm{Loading} \mathrm{behavior} \mathrm{of} \mathrm{\{ chitosan/hyaluronic}$ acid\}(n) layer-by-layer assembly films toward myoglobin: an electrochemical study. J Phys Chem B. 2006;110:23710-8.

13. Couto DS, Alves NM, Mano JF. Nanostructured multilayer coatings combining chitosan with bioactive glass nanoparticles. J Nanosci Nanotechnol. 2009;9:1741-8.
14. Tabrizian M. Biorecognition through layer-by-layer polyelectrolyte assembly: in-situ hybridization on living cells. Biomacromolecules. 2006;7:2742-50.

15. Castelnovo M, Joanny JF. Formation of polyelectrolyte multilayers. Langmuir. 2000;16:7524-32.

16. Picart C, Schneider A, Etienne O, Mutterer J, Schaaf P, Egles C, et al. Controlled degradability of polysaccharide multilayer films in vitro and in vivo. Adv Funct Mater. 2005;15:1771-80.

17. Sukhorukov GB, Donath E, Lichtenfeld H, Knippel E, Knippel M, Budde A, et al. Layer-by-layer self assembly of polyelectrolytes on colloidal particles. Colloids Surf A. 1998;137:253-66.

18. Schonhoff M. Layered polyelectrolyte complexes: physics of formation and molecular properties. J Phys Condens Matter. 2003; 15:R1781-808.

19. Goddard C. Handbook of nanoscience, engineering, and technology. Boca Raton, FL: CRC Press; 2003.

20. Caruso F. Nanoengineering of particle surfaces. Adv Mater. 2001;13:11-22.

21. Qiu XP, Leporatti S, Donath E, Mohwald H. Studies on the drug release properties of polysaccharide multilayers encapsulated ibuprofen microparticles. Langmuir. 2001;17:5375-80.

22. Anal AK, Stevens WF. Chitosan-alginate multilayer beads for controlled release of ampicillin. Int J Pharm. 2005;290:45-54.

23. Feng Q, Zeng GC, Yang PH, Wang CX, Cai JY. Self-assembly and characterization of polyelectrolyte complex films of hyaluronic acid/chitosan. Colloids Surf A. 2005;257:85-8.

24. Lee SB, Lee YM, Song KW, Park MH. Preparation and properties of polyelectrolyte complex sponges composed of hyaluronic acid and chitosan and their biological behaviors. J Appl Polym Sci. 2003;90:925-32.

25. Schneider A, Vodouhe C, Richert L, Francius G, Le Guen E, Schaaf P, et al. Multifunctional polyelectrolyte multilayer films: combining mechanical resistance, biodegradability, and bioactivity. Biomacromolecules. 2007;8:139-45.

26. Kim SJ, Shin SR, Lee KB, Park YD, Kim SI. Synthesis and characteristics of polyelectrolyte complexes composed of chitosan and hyaluronic acid. J Appl Polym Sci. 2004;91:2908-13.

27. Wang CY, Ye SQ, Dai L, Liu XX, Tong Z. Enhanced resistance of polyelectrolyte multilayer microcapsules to pepsin erosion and release properties of encapsulated indomethacin. Biomacromolecules. 2007;8:1739-44.

28. Dini E, Alexandridou S, Kiparissides C. Synthesis and characterization of cross-linked chitosan microspheres for drug delivery applications. J Microencapsul. 2003;20:375-85.

29. Bertrand P, Jonas A, Laschewsky A, Legras R. Ultrathin polymer coatings by complexation of polyelectrolytes at interfaces: suitable materials, structure and properties. Macromol Rapid Commun. 2000;21:319-48.

30. Orive G, Hernandez RM, Gascon AR, Igartua M, Rojas A, Pedraz JL. Microencapsulation of an anti-VE-cadherin antibody secreting 1B5 hybridoma cells. Biotechnol Bioeng. 2001;76:285-94.

31. Orive G, Hernandez RM, Gascon AR, Igartua M, Pedraz JL. Development and optimisation of alginate-PMCG-alginate microcapsules for cell immobilisation. Int J Pharm. 2003;259:57-68.

32. Grech JMR, Mano JF, Reis RL. Chitosan beads as templates for layer-by-layer assembly and their application in the sustained release of bioactive agents. J Bioact Compat Polym. 2008;23: 367-80.

33. Mo Y, Takaya T, Nishinari K, Kubota K, Okamoto A. Effects of sodium chloride, guanidine hydrochloride, and sucrose on the viscoelastic properties of sodium hyaluronate solutions. Biopolymers. 1999;50:23-34.

34. Kim DK, Han SW, Kim CH, Hong JD, Kim K. Morphology of multilayers assembled by electrostatic attraction of oppositely charged model polyelectrolytes. Thin Solid Films. 1999;350: 153-60. 
35. Ye SQ, Wang CY, Liu XX, Tong Z. Multilayer nanocapsules of polysaccharide chitosan and alginate through layer-by-layer assembly directly on PS nanoparticles for release. J Biomater Sci Polym Ed. 2005;16:909-23.

36. Gupta KC, Kumar MNVR. Preparation, characterization and release profiles of $\mathrm{pH}$-sensitive chitosan beads. Polym Int. 2000;49: $141-6$.

37. Gupta KC, Jabrail FH. Glutaraldehyde and glyoxal cross-linked chitosan microspheres for controlled delivery of centchroman. Carbohyd Res. 2006;341:744-56.

38. Vasiliu S, Popa M, Rinaudo M. Polyelectrolyte capsules made of two biocompatible natural polymers. Eur Polym J. 2005;41:923-32.

39. Mano JF. Stimuli-responsive polymeric systems for biomedical applications. Adv Eng Mater. 2008;10:515-27.

40. Prabaharan M, Mano JF. Stimuli-responsive hydrogels based on polysaccharides incorporated with thermo-responsive polymers as novel biomaterials. Macromol Biosci. 2006;6:991-1008.
41. Shi J, Alves NM, Mano JF. Chitosan-alginate multilayer beads containing poly(N-isopropylacrylamide) for dual-stimuli-responsive drug release. J Biomed Mater Res B. 2008;84B:595-603.

42. Richert L, Lavalle P, Payan E, Shu XZ, Prestwich GD, Stoltz JF, et al. Layer by layer buildup of polysaccharide films: physical chemistry and cellular adhesion aspects. Langmuir. 2004;20:448-58.

43. Kato N, Schuetz P, Fery A, Caruso F. Thin multilayer films of weak polyelectrolytes on colloid particles. Macromolecules. 2002;35: 9780-7.

44. Van Raamsdonk JM, Chang PL. Osmotic pressure test: a simple, quantitative method to assess the mechanical stability of alginate microcapsules. J Biomed Mater Res. 2001;54:264-71. 